\title{
Quality of life and depression in patients with irritable bowel syndrome
}

\author{
Magdalena Kopczyńska ${ }^{1}$, Łukasz Mokros², Tadeusz Pietras², Ewa Małecka-Panas ${ }^{1}$ \\ ${ }^{1}$ Department of Digestive Tract Diseases, Medical University of Lodz, Lodz, Poland \\ 2Department of Clinical Pharmacology, Medical University of Lodz, Lodz, Poland
}

Gastroenterology Rev 2018; 13 (2): 102-108

DOI: https://doi.org/10.5114/pg.2018.75819

Key words: irritable bowel syndrome, quality of life, depression.

Address for correspondence: Prof. Ewa Małecka-Panas MD, PhD, Department of Digestive Tract Diseases, Medical University of Lodz, Barlicki Memorial Hospital, 22 Kopcińskiego St, 90-153 Lodz, Poland, phone: +48 4267766 64, fax: +48 426786480 , e-mail: ewuncia@poczta.onet.pl

\begin{abstract}
Introduction: While irritable bowel syndrome (IBS) is not life-threatening for most, it has enormous influence on quality of life $(\mathrm{QOL})$ and mental health.

Aim: To evaluate the association between QOL and depressive symptoms in IBS patients.

Material and methods: A total of 87 patients and 56 healthy subjects were enrolled consecutively. All participants were asked to complete self-administered questionnaires: an IBS-specific quality of life (IBS-QOL) questionnaire and the Beck Depression Inventory questionnaire (BDI).

Results: Mean BDI score was higher in IBS group than in controls. IBS-QOL score was significantly lower in IBS patients compared to the control group, in all IBS-QOL domains: dysphoria (DY), interference with activity (IN), body image (BI), health worry (HW), food avoidance (FA), social relation (SR), and sexual (SX) and relationship (RL) issues. QOL DY, IN, and BI scores and overall score were significantly lower in women with IBS compared to men. We found that BDI score was significantly negatively correlated with IBS-QOL score in the domain of DY, IN, HW, FA, SR, and RL scores and overall QOL score. We also found a negative correlation between older age and health worry. RL and IN QOL scores represented the highest correlation with BDI score.

Conclusions: Irritable bowel syndrome is connected with impaired patient quality of life and high prevalence of depression with high correlation rate of both parameter scores. Assessment of depression and QOL should be provided during patient visits in outpatients clinics.
\end{abstract}

\section{Introduction}

Irritable bowel syndrome (IBS) is a common functional disorder of the gastrointestinal tract, characterized by abdominal pain with diarrhea and/or constipation $[1,2]$.

Gastrointestinal disorders are common in the general population - IBS affects around $10-20 \%$ of the population; however, only about $35 \%$ of them are likely to seek medical advice due to their symptoms [3, 4] The diagnosis of IBS should be based on clinical history, physical examination, minimal laboratory tests, and, when clinically indicated, a colonoscopy or other appropriate tests. To help establish the diagnosis of IBS, the Rome Criteria are used. In 2016, the IV Rome criteria were introduced. They include: symptoms of abdominal pain at least 1 day per week during the past 3 months associated with two or more of the following: 1) related to defecation, 2) associated with change in frequency of stool, and 3) associated with a change in form of stool. In contrast to Rome Criteria III, which were used for the past decade, the term "discomfort" has been eliminated from the last definition, and the phrase "improvement with defecation" was modified into "related to defecation". Similarly, the word "onset" from "associated with a change in frequency/form of stool" was deleted [2].

Because of the unknown and complicated pathomechanism of the disease, there is no radical treatment, and medical intervention is based on relieving the symptoms [5]. While IBS is not life-threatening for most, it has an enormous influence on quality of life (QOL) and mental health [6].

It is estimated that more than a half of IBS patients suffer from psychological disorders, which impede treat- 
ment and decrease QOL [7]. Depression and anxiety disorder occur in $38-100 \%$ of patients [8-11]. Because in IBS there is no specific biological disease marker, the most important criterion to determine the level of recovery is quality of life assessment [12]. Even if measures of disease activity, symptoms, and duration are available based on laboratory, imaging, or endoscopic data, they do not always correlate with wellbeing in chronic gastrointestinal disease, as is shown in patients with chronic pancreatic disease and in the elderly $[13,14]$.

The most well-known, popular, and validated specific measure of health related QOL in IBS patients is the Irritable Bowel Syndrome-Quality of Life (IBS-QOL) questionnaire invented by D.L. Patrick and D.A. Drossman. It is a self-report quality of life measure, which includes domains such as dysphoria (DY), interference with activity (IN), body image (BI), health worry (HW), food avoidance (FA), social relation (SR), and sexual (SX) and relationship (RL) issues [12].

To assess the severity of depression symptoms the Beck Depression Inventory (BDI) was used. The Polish version of the BDI was elaborated and validated by Parnowski and Jernajczyk [15]. Both of the tests are easy to conduct and may be performed during the patient's visit to an outpatient clinic. Health-related quality of life and depression recognition may help improve the management of IBS. The relation between the level of depression and quality of life impairment in IBS is poorly understood.

\section{Aim}

The aim of the present study was to evaluate the QOL in patients with IBS and to estimate the frequency of depression in this group. In addition, we determined the correlation between depression and QOL in IBS patients.

\section{Material and methods}

Patients with IBS were recruited from the Outpatient Clinic of the Department of Digestive Tract Diseases, Medical University of Lodz, Poland. The diagnosis of IBS was based on the III Rome Criteria. Because of the recent introduction of the IV Rome Criteria, patients were assessed once again and all of patients fulfilled new criteria. The study comprised 87 IBS patient: 18 (20.69\%) males and 69 (79.31\%) females, aged 18-60 years (mean: $39.22 \pm 11.84)$. The control group consisted of 56 healthy volunteers: 17 (30.36\%) males and 39 (69.64\%) women aged 18-60 years (mean: 37.0536 $\pm 12.74)$. No significant comorbidities were noted in the study group.

IBS-QOL and BDI questionnaires as well as demographic data and clinical data were collected. There were no significant differences in gender $(p=0.19)$, age $(p=0.30)$, body mass index (BMI) $(p=0.87)$, work activity $(p=0.23)$, practiced profession $(p=0.16)$, marital status $(p=0.19)$, and financial situation $(p=0.71)$ between the two groups. Mean duration of illness in IBS patients was 7.46 years. In the IBS group the number of IBS-constipation patients was 35 (41.18\%), IBS diarrhea was 29 (34.12\%), and IBS-mixed was 21 (24.71\%).

The study was carried out in accordance with the Helsinki Declaration. The study protocol was approved by the Local Ethics Committee of the Medical University of Lodz.

IBS-QOL was measured using the Polish version of the IBS-QOL questionnaire. The IBS-QOL contains 34 questions. A five-point Likert scale was used to assess how much the statement described the feelings of the respondent: not at all, slightly, moderately, quite a bit, and extremely or a great deal. The IBS-QOL is transformed into a $0-100$ scale, where 0 represents minimum QOL and 100 represents maximum QOL.

Depression was measured with the Beck Depression Inventory (BDI). It is a self-rating questionnaire, which consists of 21 items. Each item is assigned a score ranging from 0 to 3, to indicate the degree of severity of the symptom, with the total score ranging from 0 to 63 . The standard cut-off scores are as follows: 0-11, lack of depression; 12-26, mild depression; 27-49, moderate depression; and 50-63, severe depression.

\section{Statistical analysis}

Nominal variables were presented as percentages or fractions. Normally distributed continuous variables were shown as mean values with standard deviations. Normality of distribution was verified with W Shapiro-Wilk test. Ordinal variables and continuous variables of distribution other than normal were presented as median values with lower and upper quartile and interquartile range (IQR). Depending on the number of observations in the groups, $\chi^{2}$ test with Yates' correction or two-sided Fisher's exact test were used to compare nominal variables. To compare continuous variables between two groups, Student's t-test (with independent variance estimation for variables of distribution other than normal) was used. In case of more than two groups, analysis of variance (ANOVA) or non-parametric Kruskal-Wallis test were performed. Association between two continuous variables was assessed with Pearson correlation quotient. All analyses were performed with Statistica 12.0 (Statsoft, Tulsa, USA). A level of $p \leq 0.05$ was considered statistically significant.

\section{Results}

Among patients with IBS, based on the BDI, depression was diagnosed more frequently than in the control 
group and concerned 38 (46.34\%) patients vs. 5 (8.93\%) controls $(p<0.0001)$. The most frequent was moderate depression, which was seen in $37(45.12 \%)$, and severe depression was observed in $1(1.22 \%)$ IBS patient. The mean score of the BDI questionnaire was $10.78 \pm 6.804$ in the IBS group vs. $4.96 \pm 0.651$ in controls ( $p<0.0001)$.

There were significant differences in IBS-QOL between IBS and control group, particularly in overall quality of life (OV), where mean IBS-QOL score was 61.62 \pm 19.88 in IBS patients compared to $98.30 \pm 4.11$ in controls ( $p<0.0001)$, as shown in Figure 1.

In addition, the mean dysphoria score (DY) in the IBS group was $59.69 \pm 24.68$, which was significantly lower compared to the control group $99.33 \pm 2.71$ $(p<0.0001)$. The mean score of interference with activity (IN) in IBS patients was $61.86 \pm 21.86$ compared to $98.02 \pm 6.09$ in controls, i.e. it was significantly lower $(p<0.0001)$. The mean score in the body image $(\mathrm{BI})$ domain in IBS patients was $59.77 \pm 24.78$ compared to $96.54 \pm 7.24$ in controls, i.e. it was significantly lower $(p<0.0001)$. Mean score of health worry (HW) in IBS patients was $58.23 \pm 20.98$ compared to $98.66 \pm 5.22$ in controls, i.e. it was significantly lower $(p<0.0001)$. Mean score of food avoidance (FA) in IBS patients was $44.25 \pm 25.58$ compared to $94.94 \pm 10.97$ in controls, i.e. it was significantly lower $(p<0.0001)$. Mean score of social relation (SR) in IBS patients was $67.52 \pm 23.82$ compared to $99.33 \pm 2.29$ in controls, i.e. it was significantly lower $(p<0.0001)$. The mean score in the sexual (SX) domain in IBS patients was $76.43 \pm 26.30 \mathrm{com}$ pared to $99.77 \pm 1.67$ in controls, i.e. it was significantly lower $(p<0.0001)$. Mean score in the relationship $(\mathrm{RL})$ domain in IBS patients was $71.74 \pm 21.20$ compared to $99.25 \pm 3.29$ in controls, i.e. it was significantly lower $(p<0.0001)$, as shown in Figure 1.
In addition, in the IBS group IBS-QOL score was significantly lower among women than in men, especially in overall IBS-QOL: $59.02 \pm 19.43$ vs. $71.65 \pm 19.43$ ( $p<0.05$ ), in the domain of DY $56.70 \pm 24.82$ vs. 71.18 \pm 20.99 ( $p<0.05)$, IN $59.11 \pm 21.02$ vs. $72.42 \pm 22.38$ $(p<0.05)$, and $\mathrm{BI} 55.07 \pm 23.15$ vs. $77.77 \pm 23.01$ ( $p<$ 0.05) (Figure 2).

In the next step of the study we measured the correlation between IBS-QOL scores (particular domains and overall QOL) and BDI score.

BDI score was significantly negatively correlated with the domain of dysphoria (DY) $(r=-0.26 ; p<0.05)$ (Figure 3), interference with activity (IN) $(r=-0.39 ; p<$ $0.05)$ (Figure 4), health worry (HW) $(r=-0.31 ; p<0.05)$ (Figure 5), food avoidance (FA) $(r=-0.27 ; p<0.05)$ (Figure 6), sexual domain (SX) $(r=-0.42 ; p<0.05)$ (Figure 7) social relation (SR) $(r=-0.38, p<0.05)$ (Figure 8), and overall QOL (OV) $(r=-0.35 ; p<0.05)$ (Figure 9). We also found a correlation between older age and health worry (HW) $(r=-0.3524, p<0.05)$ (Figure 10). The domains of sexual (SX) and interference with activity (IN) represented the highest correlation with BDI score.

\section{Discussion}

Despite IBS is a non-life-threatening disorder, associated with decreased life expectancy; it has an important impact on patients' lives and the healthcare system. In this study, we confirmed that patients with IBS have higher prevalence of depression and lower quality of life compared to healthy controls.

In our study the prevalence of reported depression was 38 (46.34\%) IBS patients vs. 5 (8.93\%) controls. In other studies the prevalence of depression was 38$100 \%$ in IBS patients [8-10, 16]. Tosic-Golubovic et al. found depression in $83.33 \%$ of IBS patients - in $13.33 \%$

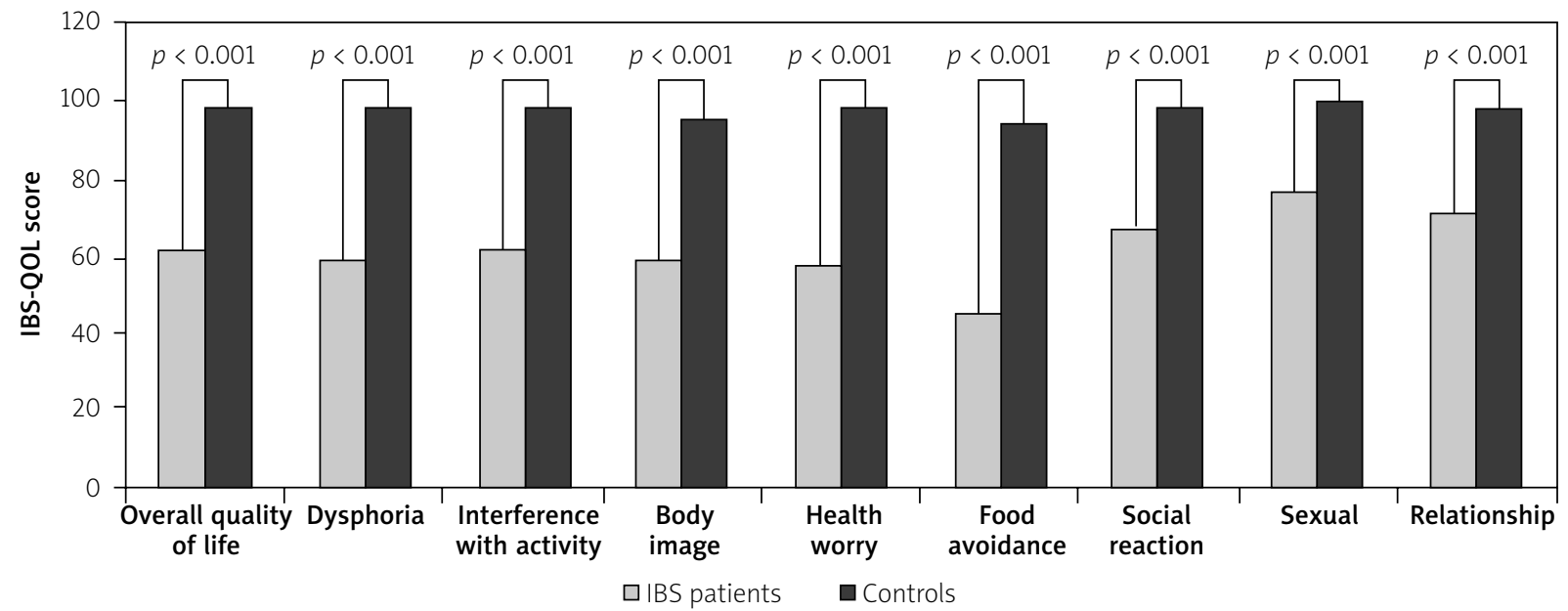

Figure 1. Comparison of quality of life domains between IBS patients and healthy controls. All differences were statistically significant 


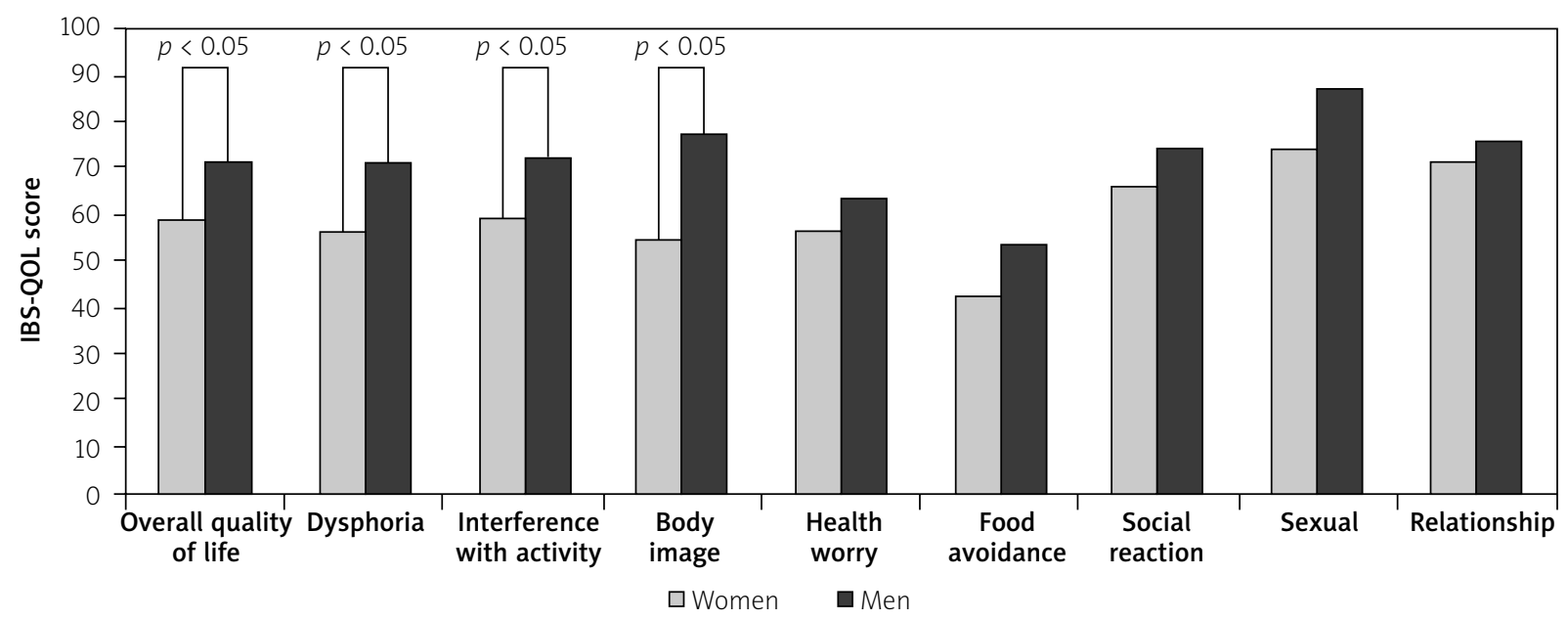

Figure 2. Comparison of quality of life domains in men and women with IBS

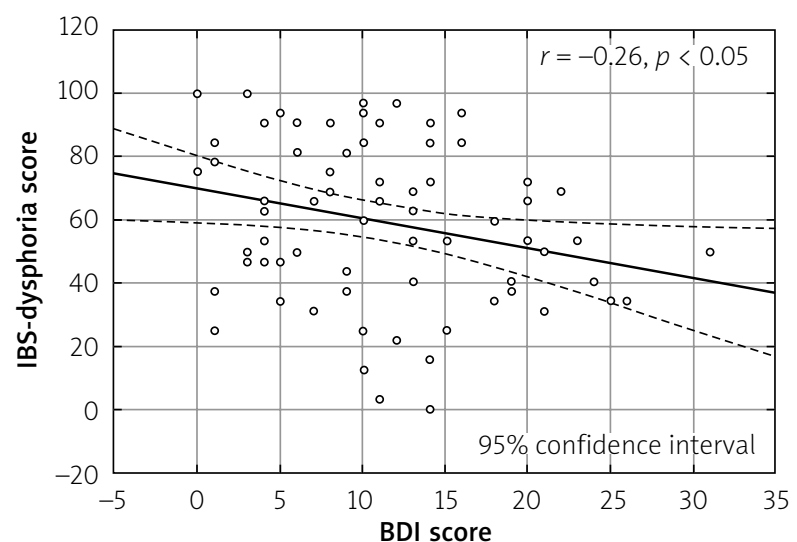

Figure 3. Correlation between BDI score and dysphoria

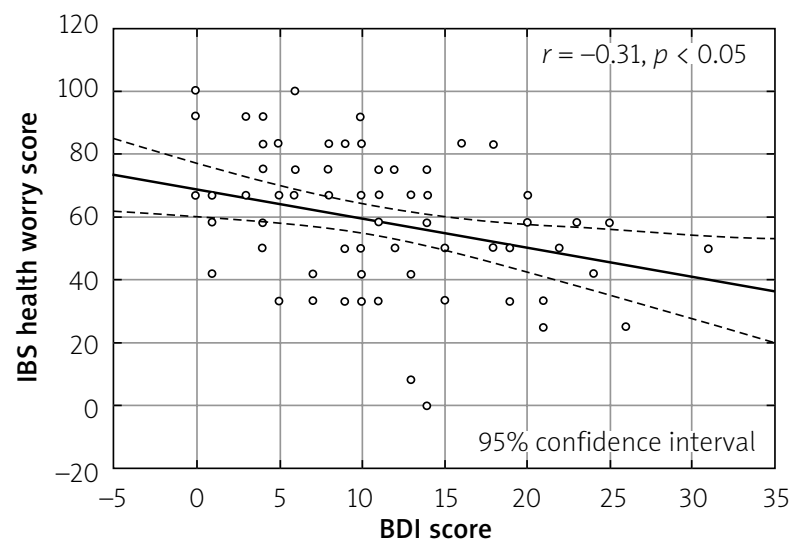

Figure 5. Correlation between BDI sore and health worry

of them even severe, which needed hospitalisation. The group of IBS patient was small and was recruited from a high-reference department, where patients with severe health problems were admitted [8].

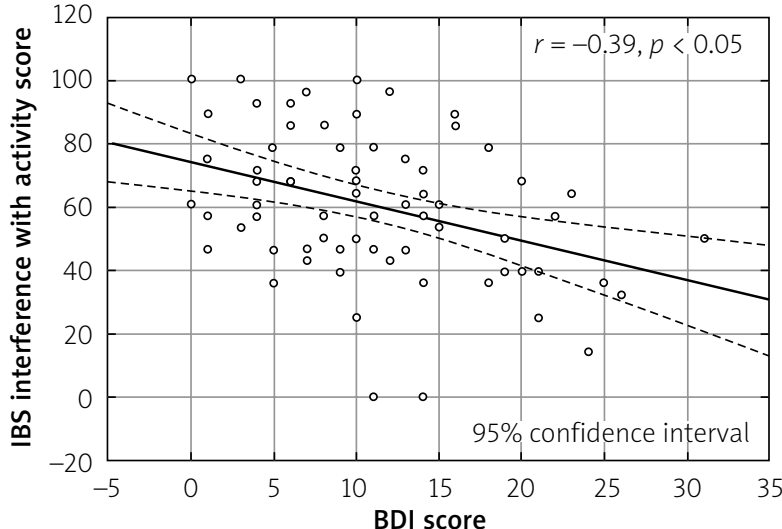

Figure 4. Correlation between BDI score and interference with activity

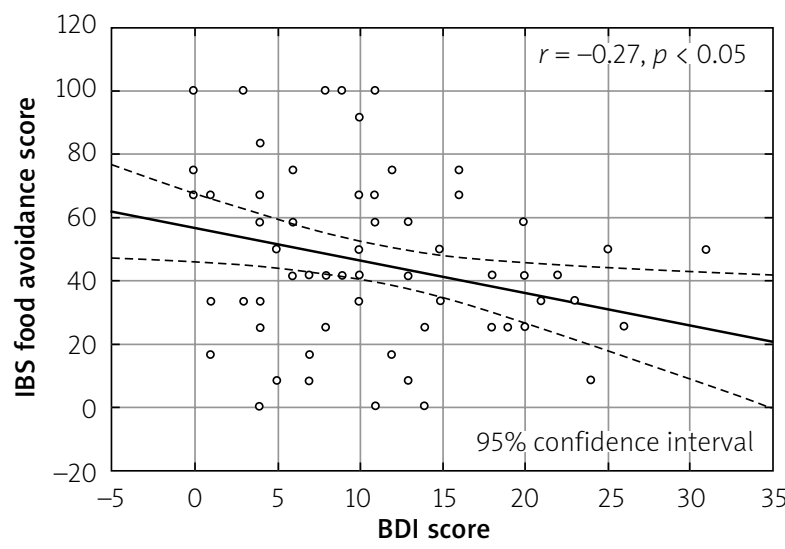

Figure 6. Correlation between BDI sore and food avoidance

We observed higher prevalence of depression in women, but the difference was not significant. The same trend has been observed previously; similar results were obtained by Björkman et al. [17], while other 


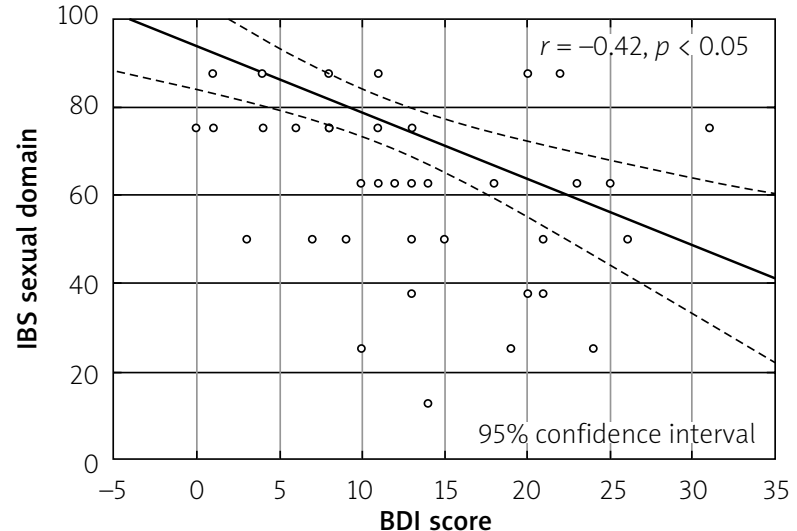

Figure 7. Correlation between BDI score and sexual domain

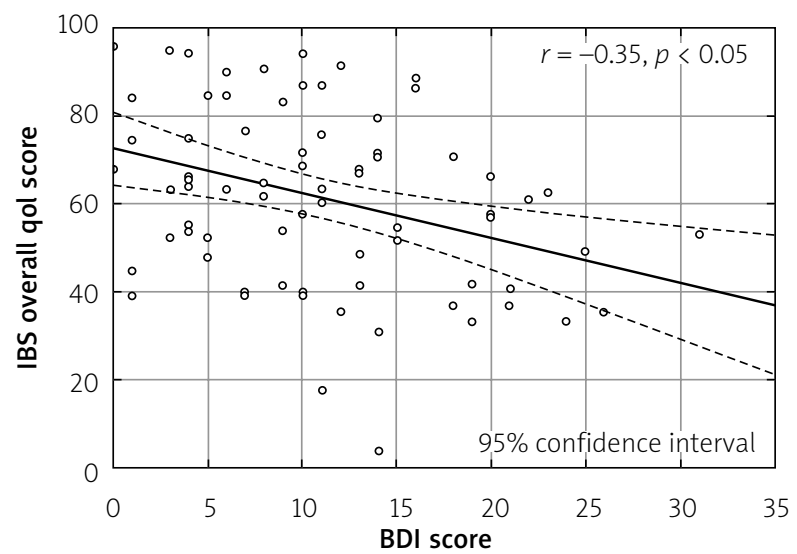

Figure 9. Correlation between BDI score and overall quality of life

authors confirmed significantly higher depression rates in IBS women compared to men [16, 18]. The gender differences observed may be due to the cultural background and lower number of men seeking medical help $[17,19]$. In addition, women have difficulties in coping with their multiple roles in society and are more exposed to stressful events and risk factors for depression during their life. This may also explain the high extraintestinal symptoms rate in women with IBS $[18,20]$.

In this study we confirmed that QOL is lower in patients with IBS than in the control group. The same observations were made in other studies. In addition, several studies revealed that QOL in IBS patients was significant lower than in patients with other chronic disease, like gastroesophageal reflux disease (GERD), end-stage kidney disease (ESRD), inflammatory bowel disease (IBD), liver disease, heart failure, diabetes, and chronic pancreatitis [21-23].

The greatest impairment in QOL was observed for the subscale of FA followed by HW, DY, BI, and IN.

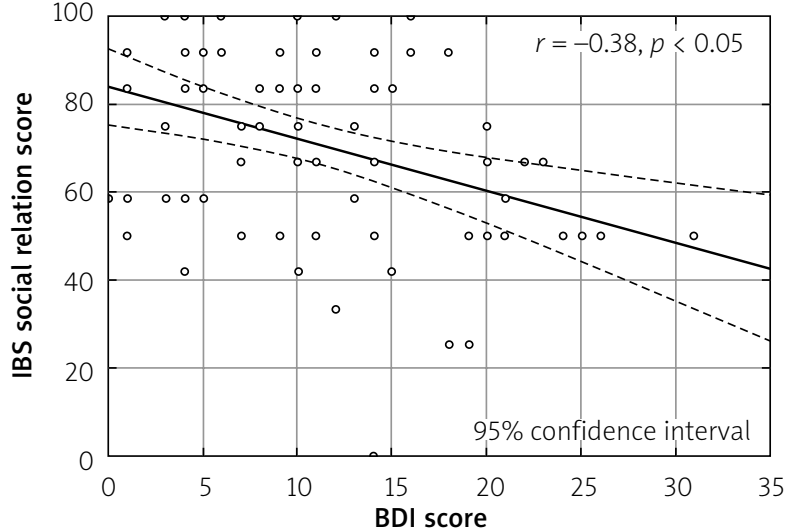

Figure 8. Correlation between BDI score and social relation

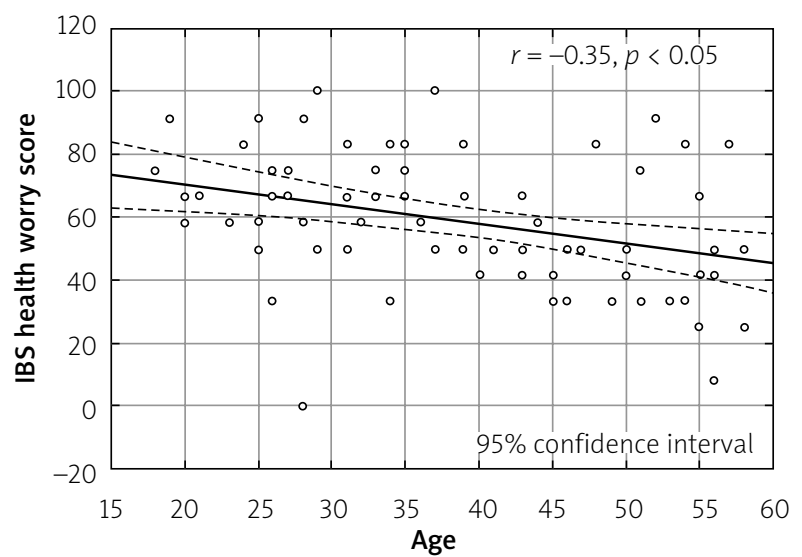

Figure 10. Correlation between age and health worry is significant

These findings were similar to those from an international survey made by Drossman et al., in which the greatest impairments of similar magnitude were shown for FA, DY, and IN but not for body image [24]. Also, Hou et al. in their multicenter study found that in IBS the most impaired domains were FA, HW, and DY. The IBS-QOL overall score was similar in Egypt, Mexico, and Poland, but higher in China, which may be due to the cultural background in different countries, e.g. race, food, beliefs, social milieu, and health-care system $[25,26]$.

Drossman et al. and Hou et al. reported less impaired quality of life in the sexual domain [24, 26]. It may be due to the fact that in most cultures patients do not willingly express their sexual activities to someone else. Sexual problems may well exist, but cultural factors may inhibit questionnaire respondents from revealing these difficulties [25].

Generally, we found lower QOL in IBS women compared to men. We found that not only total IBS-QOL 
score was significantly lower in females, but also for particular domains, such as: dysphoria, body image, and interference with activity. Tang et al. observed the same gender differences in dysphoria and body image but also found significant differences between men and woman in health worry, social, and sexual domains. In contrast, Zhu et al. found in IBS-diarrhea significantly impaired QOL in domains: food avoidance and social relation. The difference may be due to the IBS group chosen (IBS-diarrhea) [17, 27]. In IBS women impaired QOL can be caused by estrogens, which may increase visceral sensitivity and can cause pain [28]. Women are also more concerned about their health than men and more frequently have somatic symptoms connected with IBS, which leads to more frequent visits in out-patients clinic [19, 29].

In our study we found negative significant correlation between total quality of life, dysphoria, interference with activity, body image, health worry, food avoidance, and sexual domains and BDI score. Other studies found similar correlations, which shows that IBS contributes to the negative impact on QOL and depression [18, 27]. Cho et al. showed that IBS patients with depression more often had serious intestinal symptoms and their QOL was compromised significantly [30]. These findings also suggest that IBS symptoms can aggravate patients' psychological burden and that comorbid depression may aggravate patients subjective feelings about their intestinal and somatic symptoms. This may lead patients to avoid particular foods and disproportionately worry about their health, reducing their outside activities, and in turn their QOL. Impairment of the patients' QOL depends significantly on depression.

Sexual and interference with activity domains represented the highest correlation with BDI score in our study. Severity of IBS symptoms and psychological factors (e.g. childhood abuse) may influence sexual dysfunction, which can decrease QOL and may influence their mental condition [31].

Irritable bowel syndrome is also known to interfere with the physical aspects of health-related QOL including daily activities and work productivity [8, 32]. Increased bowel movement frequency could understandably limit the ability of patients to go out and thus engage in daily activities like work, travel, and other social/leisure activities [33]. Avoidance of any activities impairs QOL and may be a risk factor of depression.

We also found negative correlation between age and health worry. Tang et al. found a similar association [29]. This is probably due to the fact that younger people feel less fear about their future life. Also, general QOL is lower in older patients [34].
The BDI score and IBS-QOL questionnaires seem to be cheap, helpful, easy-to-use instruments to screen for depression and $\mathrm{QOL}$ in these patients. A multidisciplinary approach addressing depression may not only improve patients' emotional well-being but also may have some additional benefits on their disease activities related to IBS [35]. Addressing the gastrointestinal symptoms alone may not improve QOL in IBS. Psychological and psychiatric treatment in some cases may contribute to the improved IBS patient well-being.

\section{Conclusions}

Our study once again shows that IBS is connected to impaired patient quality of life and high prevalence of depression, with high correlation between those two variables. We speculate that IBS symptoms contribute to low quality of life in those patients, which may cause depression traits in some of them. Assessment of depression and health-related QOL should be provided during patients' visits to outpatient clinics.

\section{Acknowledgments}

Project supported by the Polish Society for the Prevention of Digestive Tract Neoplasms and a grant for young scientists of the Medical University of Lodz, number 502-03/1-002-01/502-14-039.

\section{Conflict of interest}

The authors declare no conflict of interest.

\section{References}

1. Longstreth GF, Thompson WG, Chey WD, et al. Functional bowel disorders. Gastroenterology 2016; 130: 1480-91.

2. Lacy BE, Mearin F, Chang L, et al. Bowel disorders. Gastroenterology 2016; 150: 1393-407.e5.

3. Kennedy TM, Jones RH, Hungin APS, et al. Irritable bowel syndrome, gastro-oesophageal reflux, and bronchial hyper-responsiveness in the general population. Gut 1998; 43: 770-4.

4. Ziółkowski BA, Pacholec A, Kudlicka M, et al. Prevalence of abdominal symptoms in the Polish population. Prz Gastroenterol 2012; 7: 20-5.

5. Fichna J, Wood JT, Papanastasiou M, et al. Endocannabinoid and cannabinoid-like fatty acid amide levels correlate with pain-related symptoms in patients with IBS-D and IBS-C: a pilot study. PLoS One 2013; 8: e85073.

6. Creed F, Ratcliffe J, Fernandes L, et al. Outcome in severe irritable bowel syndrome with and without accompanying depressive, panic and neurasthenic disorders. Br J Psychiatry 2005; 186: 507-15.

7. Hillilä MT, Siivola MT, Färkkilä MA. Comorbidity and use of health-care services among irritable bowel syndrome sufferers. Scand J Gastroenterol 2007; 42: 799-806. 
8. Tosic-Golubovic S, Miljkovic S, Nagorni A, et al. Irritable bowe syndrome, anxiety, depression and personality characteristics. Psychiatr Danub 2010; 22: 418-24.

9. Hausteiner-Wiehle C, Henningsen P. Irritable bowel syndrome: relations with functional, mental, and somatoform disorders. World J Gastroenterol 2014; 20: 6024-30.

10. Woodman CL, Breen K, Noyes R Jr, et al. The relationship between irritable bowel syndrome and psychiatric illness. Psychosomatics 2016; 39: 45-54

11. Kopczyńska M, Wrona E, Małecka-Panas E. Zaburzenia depresyjne w zespole jelita drażliwego. Terapia 2015; 3: 19-23.

12. Patrick DL, Drossman DA, Frederick IO, et al. Quality of life in persons with irritable bowel syndrome (development and validation of a new measure). Dig Dis Sci 1998; 43: 400-11.

13. Mokrowiecka A, Pinkowski D, Malecka-Panas E, Johnson CD. Clinical, emotional and social factors associated with quality of life in chronic pancreatitis. Pancreatology 2010; 10: 39-46.

14. Bartoszek A, Domżał-Drzewicka R, Kachaniuk $H$, et al. The state of nutrition and the self-assessment of symptoms of depression in the group of seniors living in the countryside of Lublin province - preliminary report. Prz Gastroenterol 2015 10: 208-14.

15. Parnowski T, Jernajczyk W. Beck's depression inventory in the rating of mood in normal subjects and in patients with affective disturbances [Polish]. Psychiatr Pol 1977; 11: 417-21.

16. Kabra N, Nadkarni A. Prevalence of depression and anxiety in irritable bowel syndrome: a clinic based study from India. Indian J Psychiatry 2013; 55: 77-80.

17. Björkman I, Jakobsson Ung E, Ringström G, et al. More similarities than differences between men and women with irritable bowel syndrome. Neurogastroenterol Motil 2015; 27: 796-804.

18. Tang Y, Yang W, Wang YL, Lin L. Sex differences in the symptoms and psychological factors that influence quality of life in patients with irritable bowel syndrome. Eur J Gastroenterol Hepatol 2012; 24: 702-7.

19. Galdas PM, Cheater F, Marshall P. Men and health help-seeking behaviour: literature review. J Adv Nurs 2005; 49: 616-23.

20. Simrén $M$, Abrahamsson $H$, Svedlund J, Björnsson ES. Quality of life in patients with irritable bowel syndrome seen in referral centers versus primary care: the impact of gender and predominant bowel pattern. Scand J Gastroenterol 2001; 36 545-52.

21. Gralnek IM, Hays RD, Kilbourne A, et al. The impact of irritable bowel syndrome on health-related quality of life. Gastroenterology 2016; 119: 654-60.

22. El-Serag HB, Olden K, Bjorkman D. Health-related quality of life among persons with irritable bowel syndrome: a systematic review. Aliment Pharmacol Ther 2002; 16: 1171-85.

23. Frank L, Kleinman L, Rentz A, et al. Health-related quality of life associated with irritable bowel syndrome: comparison with other chronic diseases. Clin Ther 2002; 24: 675-89.

24. Drossman DA, Morris CB, Schneck S, et al. International survey of patients with IBS: symptom features and their severity, health status, treatments, and risk taking to achieve clinical benefit. J Clin Gastroenterol 2009; 43: 541-50.

25. Jafari P, Asadollahi Z, Moini M, Seyed Mirzaie M. Health related quality of life in Iranian patients with irritable bowel syndrome: reliability and validity of the Persian versin of the IBS-OOL. Iran Red Crescent Med J 2013; 15: 723-8.
26. Hou X, Chen S, Zhang Y, et al. Quality of life in patients with irritable bowel syndrome (IBS), assessed using the IBS-quality of life (IBS-QOL) measure after 4 and 8 weeks of treatment with mebeverine hydrochloride or pinaverium nromide: results of an International Prospective Observa. Clin Drug Investig 2014; 34: 783-93.

27. Zhu L, Huang D, Shi L, et al. Intestinal symptoms and psychological factors jointly affect quality of life of patients with irritable bowel syndrome with diarrhea. Health Qual Life Outcomes 2015; 13: 49.

28. Mulak A, Taché $Y$, Larauche $M$. Sex hormones in the modulation of irritable bowel syndrome. World J Gastroenterol 2014; 20: 2433-48.

29. Tang YR, Yang WW, Liang ML, et al. Age-related symptom and life quality changes in women with irritable bowel syndrome. World J Gastroenterol 2012; 18: 7175-83.

30. Cho HS, Park JM, Lim CH, et al. Anxiety, depression and quality of life in patients with irritable bowel syndrome. Gut Liver 2011; 5: 29-36.

31. Sansone RA, Sansone LA. Irritable bowel syndrome: relationships with abuse in childhood. Innov Clin Neurosci 2015; 12: 34-7.

32. Johannesson E, Simren $M$, Strid $H$, et al. Physical activity improves symptoms in irritable bowel syndrome: a randomized controlled trial. Am J Gastroenterol 2011; 106: 915-22.

33. Singh P, Staller K, Barshop K, et al. Patients with irritable bowel syndrome-diarrhea have lower disease-specific quality of life than irritable bowel syndrome-constipation. World J Gastroenterol 2015; 21: 8103-9.

34. Motl RW, McAuley E. Physical activity, disability, and quality of life in older adults. Phys Med Rehabil Clin N Am 2010; 21 : 299-308.

35. Mahvi-Shirazi M, Fathi-Ashtiani A, Rasoolzade-Tabatabaei SK, Amini M. Irritable bowel syndrome treatment: cognitive behavioral therapy versus medical treatment. Arch Med Sci 2012; 8. $123-9$

Received: 26.06 .2017

Accepted: 30.08 .2017 